\title{
Interactions of Spectrin in Hereditary Elliptocytes Containing Truncated Spectrin $\beta$-chains
}

\author{
S. W. Eber, ${ }^{\star}$ S. A. Morris, ${ }^{\ddagger}$ W. Schröter, ${ }^{\star}$ and W. B. Gratzer ${ }^{\ddagger}$ \\ *Department of Pediatrics, University of Göttingen, 3400 Göttingen, West Germany; ${ }^{\ddagger}$ Medical Research Council Cell Biophysics Unit, \\ King's College, London WC2B 5RL, United Kingdom
}

\section{Abstract}

An abnormal spectrin, in which one subunit is truncated, has been detected in a large German family. The inheritance is autosomal dominant. The affected members of the family suffer in widely varying degree from a microcytic hemolytic anemia. The red cell morphology varies correspondingly from smooth elliptocytes to predominantly poikilocytes. The abnormal spectrin makes up $\sim 30 \%$ of the total and is almost entirely present as the dimer. The truncated chain is not phosphorylated by the endogenous CAMP-independent kinase, and it has been identified as a chain of $\beta$-type, using monoclonal antibodies. Because a univalent terminal spectrin $\alpha$-chain fragment will bind to normal dimers with an association constant lower by only a factor of two than that for the self-association of the dimers, it would be expected that the mutant dimers $\left(\alpha \beta^{\prime}\right)$ would readily enter into an association with normal $(\alpha \beta)$ dimers to give $\alpha_{2} \beta \beta^{\prime}$ tetramers (though not with each other). In dilute solution this is indeed observed, and the diminution in tetramer concentration when $30 \%$ of normal spectrin is replaced by $\alpha \beta^{\prime}$ dimers, amounts to only a small proportion. Moreover, in the membrane skeleton, if there is pairwise apposition of dimer units, only $9 \%$ of pairings will be between units that cannot associate. We have shown that the failure of $\alpha \beta^{\prime}$ dimers to enter into heterologous associations in situ is not due to the elimination of the ankyrin binding site near the truncated end of the $\beta$-chain: this site is fully functional, as judged by rebinding to spectrin-depleted vesicles. When the spectrin is extracted from the membrane in the cold, the material released initially consists almost entirely of $\alpha \beta^{\prime}$ dimers; when the spectrin of normal membranes is partly dissociated to dimers in situ by warming at low ionic strength, extraction in the cold then leads similarly to much more rapid release of the dimer than of the tetramer. The similar rates of liberation of normal and abnormal dimer make it unlikely that the interaction of the latter with the membrane is in any way defective. When mixtures of $\alpha \beta$ and $\alpha \beta^{\prime}$ dimers are bound to spectrindepleted inside-out membrane vesicles from normal cells and tetramers are allowed to form by equilibration at $30^{\circ} \mathrm{C}$, the proportion of the abnormal species appearing in the tetramer is much lower than would be expected on a statistical basis. The

Address reprint requests to Dr. Gratzer, Medical Research Council Cell Biophysics Unit, King's College, 26-29 Drury Lane, London WC2B 5RL, UK

Received for publication 29 October 1986 and in revised form 10 August 1987.

J. Clin. Invest.

(c) The American Society for Clinical Investigation, Inc.

0021-9738/88/02/0523/08 \$2.00

Volume 81, February 1988, 523-530 relation of the self-association equilibrium on the membrane to that of spectrin in dilute solution is analyzed.

\section{Introduction}

Several genetically distinct hemolytic anemias have been shown to result from anomalies in the major protein of the membrane skeleton, spectrin (see reference 1 for review). A number of these are characterized by a defect in the self-association of the spectrin dimer, which constitutes the basic structural unit, and in situ normally forms tetramers by head-tohead association, as well as a smaller proportion of higher oligomers $(2,3)$. Such effects occur in hereditary elliptocytosis and hereditary pyropoikilocytosis, and most often arise from a defect in the terminal region of the spectrin $\alpha$-chain. In what is evidently a rarer type of elliptocytosis, defective self-association results from a truncated $\beta$-chain; this has so far been seen in only two families $(6,7)$. The deletion amounts to a chain segment of molecular weight $\sim 15,000$, and it includes the sites at which the $\beta$-chain is phosphorylated by the endogenous cAMP-independent kinase. The observations suggested that the abnormal $\beta$-chains (termed $\beta^{\prime}$ ) occurred almost entirely in the form of a dimer, $\alpha \beta^{\prime}$ ), even though the remaining binding site should be capable of interaction with a normal dimer as partner; it is known for example that a univalent fragment of normal spectrin, comprising the $\alpha$-chain terminal domain, is capable of associating strongly with a normal dimer (8-10).

We have studied the properties of the spectrin from the cells of another family showing a similar $\beta$-chain mutation; we have carried out a quantitative analysis of its self-association and its attachment to the membrane, and related the results to the functional consequences of the anomaly.

\section{Methods}

Patients. The genealogy of the patients, who belong to a large German family, is shown in Fig. 1. A hematological profile is given in Table $I$. Two male members of the family (B-IV.11 and B-V.9) suffered from recurrent hemolytic crises, which necessitated occasional transfusions. After splenectomy both showed a persisting compensated microcytic hemolytic anemia. Their red cells displayed elliptocytosis and poikilocytosis (Fig. $2 b$ ). One female member of the family (B-V.10) required an exchange transfusion because of severe hyperbilirubinemia shortly after birth. Thereafter she suffered from marked hemolytic anemia and splenectomy was advised. The hemolytic anemia was rather milder in a 75-yr-old woman (B-III4), who underwent cholecystectomy because of pigment gallstones. The peripheral blood smears of both female patients showed a uniform elliptocytosis (Fig. $2 a$ ). Three other members of the family (B-II.1, B-II.5 and B-III.2) had anemia and recurring icterus, but were otherwise well. There was also one neonatal death, due to perinatal stress, arising mainly from eclampsia in the mother. A contribution to the illness was a precocious and severe hemolytic hyperbilirubinemia. 


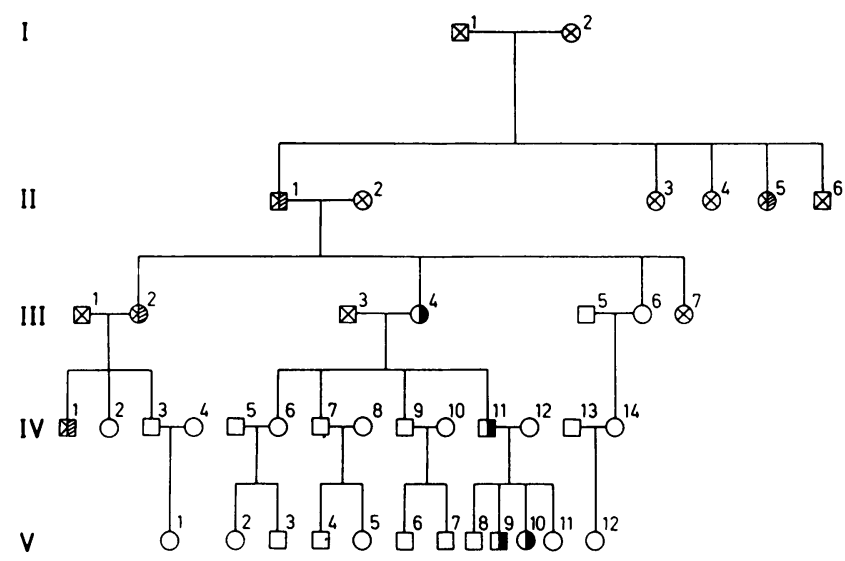

Figure 1. Genealogy of family. Squares, male, circles, female; cross, deceased. Presence of the abnormal spectrin is denoted by half-filled symbols. Half-hatched symbols refer to deceased members of the family, who were probably affected.

Severely affected members of the family (IV.11 and V.9) showed increased osmotic fragility (11) of the red cells. Thermal stability profiles (12) of the red cells of the patients revealed onset of crenation and budding at $46^{\circ} \mathrm{C}$, whereas control normal cells retained their discoid shape up to $48-49^{\circ} \mathrm{C}$. As judged by cellulose acetate strip electrophoresis, there was no abnormal hemoglobin. The total spectrin content per cell was determined by SDS-gel electrophoresis and densitometry after dissolving a volume of packed cells, containing a known number of cells (Coulter counter; Coulter Electronics, Hialeah, FL) in $10 \%$ SDS (13).

Extraction and purification of spectrin. Total spectrin was extracted from the membranes of hypotonically lysed cells (14) by incubation at low ionic strength at $36^{\circ} \mathrm{C}$, and purified by chromatography on Sepharose 4B (15). A fraction enriched in the abnormal $\left(\alpha \beta^{\prime}\right)$ dimer was obtained by extraction at low ionic strength by dialysis overnight at $4^{\circ} \mathrm{C}(14)$, followed by chromatography on Sepharose $4 \mathrm{~B}$ as before: the dimer fraction contained a large proportion of the anomalous spectrin. The composition of spectrin, liberated at $4^{\circ} \mathrm{C}$, as a function of extraction time was measured as to dimer: tetramer ratio by agarose gel electrophoresis and densitometry or by sucrose gradient centrifugation $\left(10^{5} \mathrm{~g}\right.$ for $\left.18 \mathrm{~h}\right)$. The dimer and tetramer fractions were analyzed for $\beta$ and $\beta^{\prime}$ content by SDS-gel electrophoresis.

Characterization of the anomalous spectrin. The truncated $\beta$ chains were initially detected by gel electrophoresis of the total membrane protein in the presence of SDS, using the discontinuous buffer

Table I. Hematological Data

\begin{tabular}{|c|c|c|c|c|c|}
\hline & \multicolumn{5}{|l|}{ Patient } \\
\hline & \multirow[b]{2}{*}{ III. $4^{*}$} & \multirow[b]{2}{*}{ IV. $11^{*}$} & \multicolumn{2}{|l|}{ V. $9^{8}$} & \multirow[b]{2}{*}{ V.10* } \\
\hline & & & $\mathbf{a}$ & b & \\
\hline Hemoglobin $(g /$ liter $)$ & 187 & 140 & 84 & 122 & 94 \\
\hline $\operatorname{MCV}\left(\mu^{3}\right)$ & 91 & 74 & 89 & 77 & 70 \\
\hline $\mathrm{MCHC}(\%)$ & 36 & 36 & 32 & 36 & 36 \\
\hline Reticulocytes $(\times 1,000 / \mu l)$ & 162 & 210 & 452 & 103 & 440 \\
\hline $\begin{array}{l}\text { Haptoglobin, }(\mathrm{mg} / \mathrm{ml}) \\
\quad \text { (normal range: } 0.7-3.2)\end{array}$ & 0.02 & 0.1 & & 0.03 & \\
\hline
\end{tabular}

* Not splenectomized.

$¥$ Values after splenectomy.

Values are given before splenectomy $(a)$ and after splenectomy $(b)$. system of Laemmli (16), with an acrylamide concentration gradient of $5-17 \%$. The proportion of dimer in the extracted spectrin was determined by gel electrophoresis on $2 \%$ agarose (17) at $4^{\circ} \mathrm{C}$ and in the absence of any denaturant. The buffer in both gel and reservoir was 0.1 M Tris, 0.1 M Bicine. The gels were evaluated after staining with Coomassie Blue R250 by densitometry (Joyce-Loebl Chromoscan), using an interference filter with a pass-band centered at $626 \mathrm{~nm}$. Areas under zone profiles were determined as described earlier (10), or by cutting out and weighing.

For examination of phosphorylation, ghosts, prepared by lysis of cells in $5 \mathrm{mM}$ sodium phosphate, $0.1 \mathrm{mM}$ EDTA, $0.02 \%$ phenylmethanesulfonyl fluoride, $\mathrm{pH} 7.6$, were incubated for $30 \mathrm{~min}$ in $10 \mathrm{mM}$ Tris, $5 \mathrm{mM}$ magnesium chloride, $0.5 \mathrm{mM}$ dithiothreitol, $1 \mathrm{mM}$ EGTA, $1 \mathrm{mM}\left[{ }^{32} \mathrm{P}-\gamma\right] \mathrm{ATP},\left(1 \mu \mathrm{Ci} \mathrm{mmol}{ }^{-1}\right.$ from Amersham Corp., $)$, pH 7.4. After gel electrophoresis of the spectrin in SDS, the gels, stained with Coomassie Blue, were scanned in the densitometer, dried, and placed in contact with preflashed $\mathrm{x}$-ray film for $2 \mathrm{~d}$ at $-70^{\circ} \mathrm{C}$. The films were developed and scanned.

To identify the truncated spectrin chain the proteins were transferred after SDS-gel electrophoresis to nitrocellulose membrane by electroblotting. The blots were incubated with a mixture of five monoclonal anti- $\beta$-chain antibodies, generously given to us by $D$. M. Shotton and S. Pittman (Department of Zoology, Oxford University). A second (rabbit anti-mouse) antibody, coupled to peroxidase (Dako, Copenhagen, Denmark) was used to locate the coupled primary antibody (18).

The dimer-tetramer equilibrium of the purified spectrin was studied at $30^{\circ} \mathrm{C}$. Normal and abnormal spectrins, mixed in different proportions, or diluted with buffer, were incubated at $30^{\circ} \mathrm{C}$ for $3 \mathrm{~h}$ and chilled in ice. Standard loadings were then applied to agarose gels, and the dimer and tetramer (with only traces of higher oligomers at the concentrations used) were quantitated as before. Most experiments with mixtures were performed at a spectrin concentration of about 1 $\mathrm{mg} / \mathrm{ml}$ and the buffer was $0.1 \mathrm{M}$ sodium chloride, $10 \mathrm{mM}$ phosphate, 1 $\mathrm{mM}$ dithiothreitol, $\mathrm{pH}$ 7.5. Concentrations were determined spectrophotometrically, taking $\mathrm{E}_{\mathrm{lcm}}^{1 \%}=10.7$ at $280 \mathrm{~nm}$ (19).

To study the binding of spectrin to the membrane, spectrin-depleted vesicles were prepared as described by Bennett (20) from the membranes of red cells that had been carefully freed of all traces of leukocytes by filtration through cellulose (21) and treated before lysis with the membrane-permeant protease inhibitor, diisopropylfluorophosphate. For rebinding assays, vesicles $(0-0.4 \mathrm{mg} / \mathrm{ml}$ total protein) were incubated with an invariant mixture of normal and abnormal spectrins $(50 \mu \mathrm{g} / \mathrm{ml})$ that has been converted to the dimeric state by incubation at $36^{\circ} \mathrm{C}$ after extraction at low ionic strength. Binding was assayed at $0^{\circ} \mathrm{C}$ and at physiological salt concentration in the medium employed by Bennett (20). After incubation for $90 \mathrm{~min}$, the vesicles were pelleted by centrifugation through a $20 \%$ sucrose column for 30 min at $39,000 \mathrm{~g}$ and the concentrations of the normal and abnormal spectrins remaining in the supernatant were determined by SDS-gel electrophoresis, followed by staining and densitometry. As a control, vesicles were inactivated by treatment at $100^{\circ} \mathrm{C}$ for $10 \mathrm{~min}$, and the binding assay was performed in the same way. Mixtures of dimers of abnormal and normal spectrins, bound to vesicles, were also allowed to associate to tetramers by incubation at $30^{\circ} \mathrm{C}$ for $4 \mathrm{~h}$. The spectrin was then extracted by dialysis at low ionic strength at $4^{\circ} \mathrm{C}$ as before and analysed by gel electrophoresis in the absence of denaturant. Zones corresponding to dimer and tetramer were cut from the gel, incubated in SDS-containing sample buffer (15) and applied directly to an SDSpolyacrylamide gel (15) to yield the proportion of abnormal spectrin in the dimer and tetramer components.

\section{Results}

Identification of the defect. The inheritance of the abnormal $\beta$-chain (apparent molecular weight $\sim 205,000$ ) is autosomal dominant (Fig. 1). In all the four cases that we examined quan- 


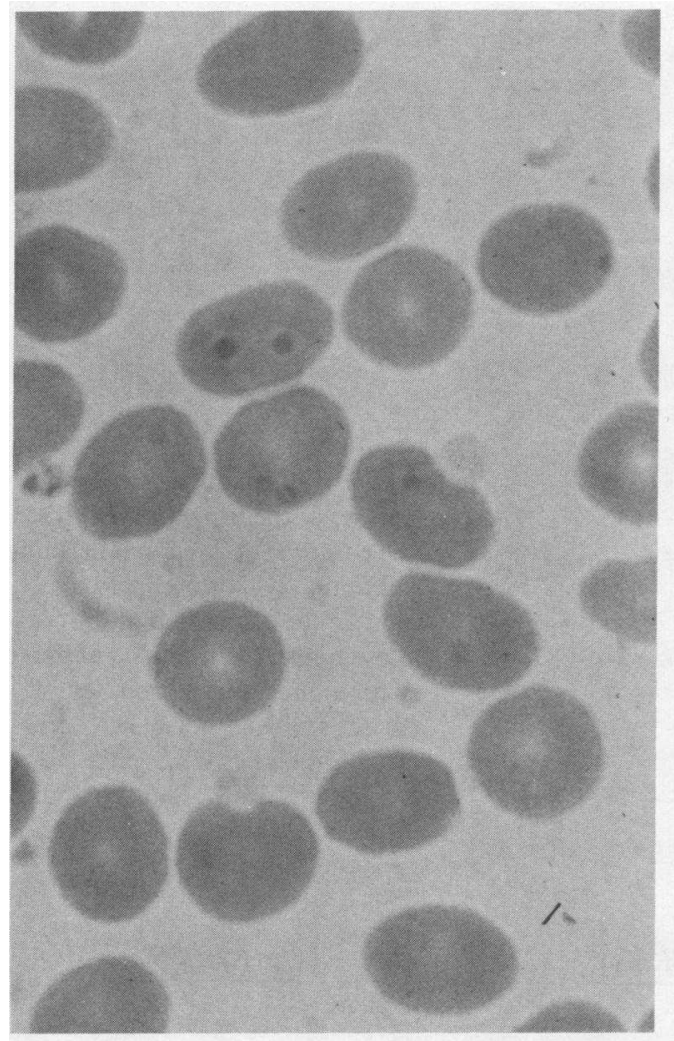

a

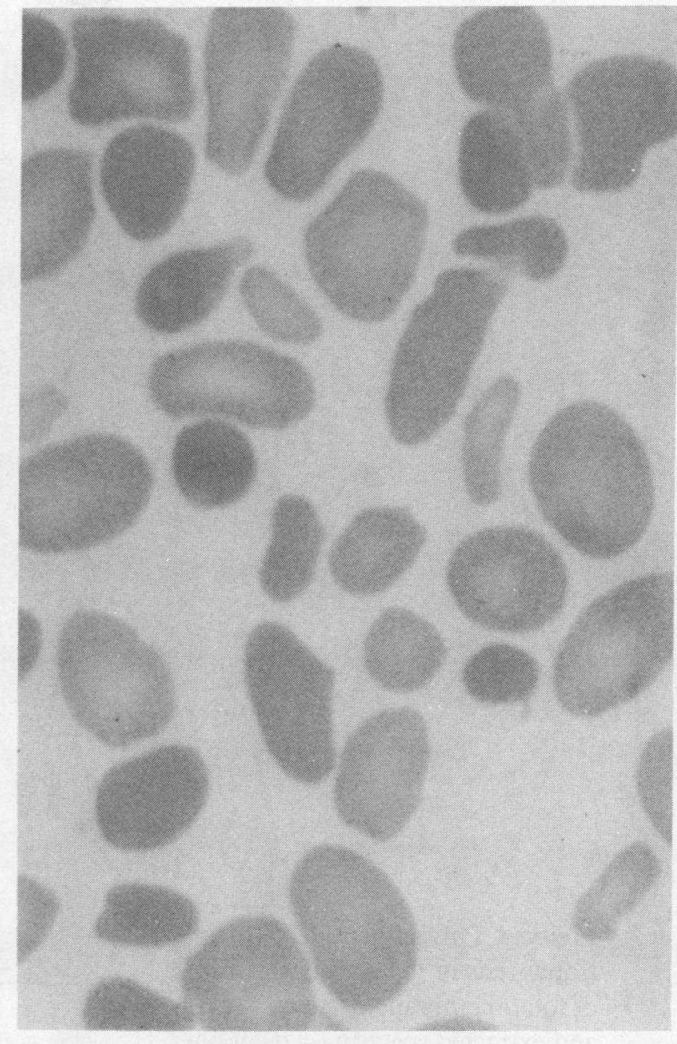

Figure 2. Blood films of patients B-V.10 (a), showing elliptocytosis, and B-IV.11 (b), showing poikilocytosis. (See text for corresponding clinical states.) titatively (Fig. 3 ) it makes up $\sim 30 \%$ of the total $\beta$-spectrin in the membrane. The third component, seen in the SDS gels and migrating ahead of the $\beta$-chain, is not subject to phosphorylation (Fig. 4). This is consistent with the deletion of a segment of the polypeptide chain at the end at which both phosphorylation and dimer self-association occur. As for the two earlier reported cases of a truncated spectrin chain $(6,7)$, the normal $\beta$-chain is reduced in amount relative to the $\alpha$-chain, and it therefore seemed likely that the mutant spectrin was a $\beta$-chain. This is now demonstrated explicitly: Fig. 5 shows that it binds anti- $\beta$-chain antibodies as effectively as the intact $\beta$-chain, whereas there is no binding of these antibodies to the $\alpha$-chain. The total spectrin content per cell was the same within experimental error as in normal erythrocytes.

Spectrin self-association. When spectrin is extracted from the membranes of the patients at $4^{\circ} \mathrm{C}, \sim 50 \%$ of the protein is recovered as a dimer (as against $<10 \%$ from normal cells), as shown by electrophoresis in agarose gels in the absence of denaturant or by chromatography on Sepharose 4B. Essentially all the abnormal spectrin is present in the dimer fraction, together with some $20 \%$ of normal spectrin. This agrees with earlier observations, and constitutes a paradox: a single $\alpha-\beta$ contact should be sufficient to allow a high degree of self-association, for a univalent fragment from the spectrin $\alpha$-chain will bind to the $\alpha \beta$ dimer with an association constant lower by a factor of only two than that for a dimer-dimer interaction $(8$, 10). The free energy of the univalent interaction at $30^{\circ} \mathrm{C}$ is
$-7.0 \mathrm{kcal} \mathrm{mol}^{-1}(10)$. Clearly, since no heterologous contacts would be available, the $\alpha \beta^{\prime}$ dimer cannot be expected to associate with itself, but the hetero-tetramer, $\alpha_{2} \beta \beta^{\prime}$, should form with an affinity not greatly less than that for the self-association of normal spectrin. This proposition has been tested by measuring the dimer-tetramer equilibrium in mixtures of $\alpha \beta$ and $\alpha \beta^{\prime}$ at constant total spectrin concentration.

In measuring the self-association of spectrin dimers one can make use of the unusually high activation energy for this process (14). Thus, whereas the system will reach equilibrium in a few minutes at $37^{\circ} \mathrm{C}$ and in minutes to hours at $25-30^{\circ} \mathrm{C}$, the reaction is effectively frozen at lower temperatures, and at $4^{\circ} \mathrm{C}$ there is no interconversion over a period of weeks. One can therefore chill an equilibrium mixture in ice and separate its components by gel electrophoresis or other methods with no reequilibration. This characteristic is preserved in the interaction with the univalent fragment (8-10), and therefore by implication with the univalent $\alpha \beta^{\prime}$ dimer.

To analyze the results, we proceed as follows: we consider the equilibria between the normal spectrin dimer, $S$, and the univalent fragment, or truncated dimer, $F$ :

$2 S \backsim S_{2} \quad$ with association constant $K_{\mathrm{s}}$

and $S+F\left\llcorner S F \quad\right.$ with association constant $K_{\mathrm{f}}$.

With the aid of the conservation conditions, viz

$\bar{S}=[S]+[S F]+2\left[S_{2}\right]$ 

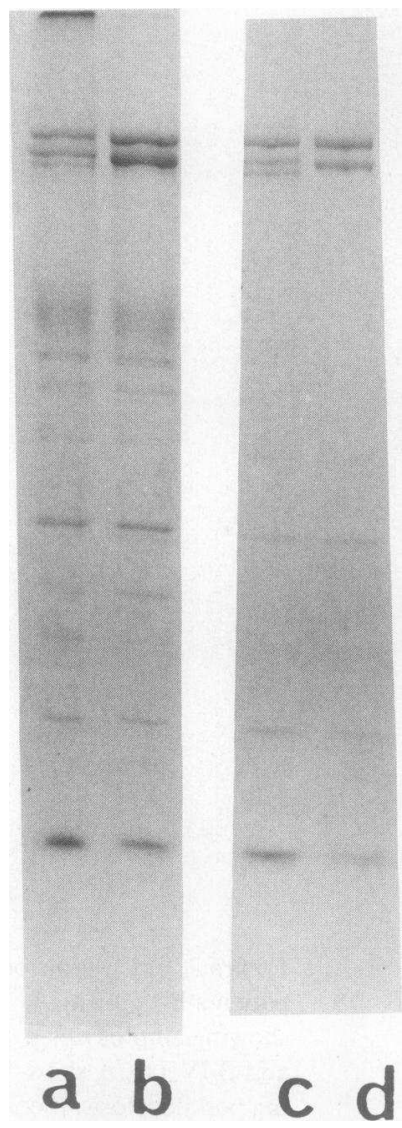

Figure 3. Polyacrylamide gel electrophoresis in the presence of SDS of total membrane protein $(a, b)$ and extracted spectrin $(c, d)$ of patient $(a, c)$ and normal subject $(b, d)$.

and

$\bar{F}=[F]+[S F]$,

(where $\bar{S}$ and $\bar{F}$ are the total molar concentrations of $S$ and $F$ in the system, expressed as dimers), and the mass-action relations, one may readily obtain the total concentration, $t$, of spectrin, present as tetramer, i.e., $2\left[S_{2}\right]+2[S F]$, as a function of $\bar{F} / C \equiv f$ (say) with $\bar{F}+\bar{S}=C$ (constant). Solving the system of simple simultaneous equations, that results from these relations, we obtain
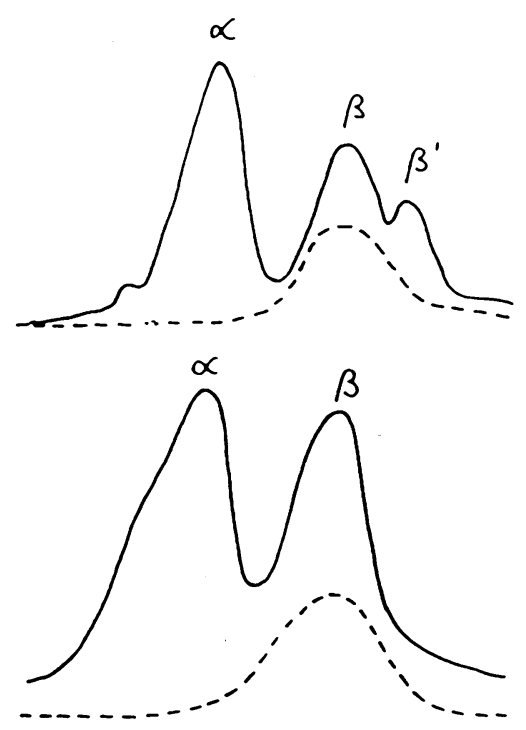

Figure 4. Densitometric scans of stained electrophoretic gel (solid line) and autoradiograph (broken line) of $\left.{ }^{[2} \mathrm{P}\right]$ phosphorylated spectrin dimer from patient $(a)$ and normal subject $(b)$. Migration from left to right.

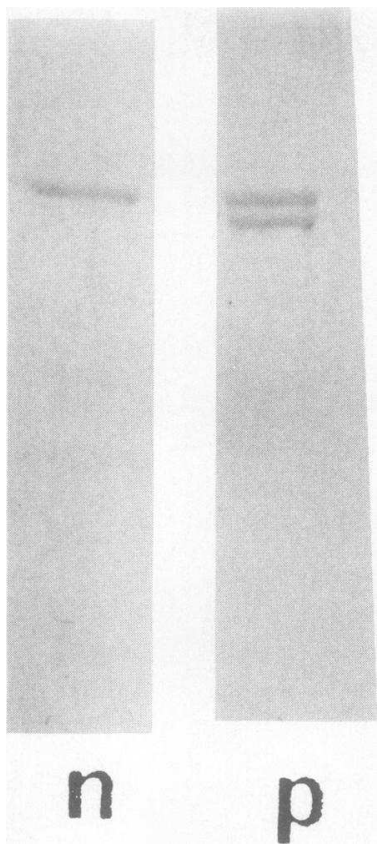

Figure 5. Immunoblot, showing identity of the truncated spectrin $\left(\beta^{\prime}\right)$ component as a $\beta$-chain. Indirect antibody labeling (peroxidase stain), after incubation of nitrocellulose transfer with mixed anti- $\beta$ chain monoclonal antibodies, of patient's $(P)$ and normal $(N)$ spectrin. (Antibodies were generously provided by D. M. Shotton and S. Pittman, University of Oxford.)

$f=\frac{C-2 K_{s} K_{f}[S]^{3}-\left(2 K_{\mathrm{s}}+K_{\mathrm{f}}\right)[S]^{2}-\left(1-K_{f} C\right)[S]}{\mathrm{C}\left(1+2 K_{\mathrm{f}}[S]\right)}$

$t=2 C(1-\alpha)-2[S]-2 K_{\mathrm{s}}[S]^{2}$.

We then calculate $f$ as a function of $[S]$ from Eq. 1 and a relation between $f$ and $t$ is obtained by employing Eq. 2 . It should be remarked that if $F$ were functionally indistinguishable from $S, t$ would be constant with change of $f$ (Fig. 6). Regardless of the value of $K_{\mathrm{f}}$, on the other hand, the concentration of tetramer will fall to zero when $f$ approaches unity, if it cannot self-associate. Fig. 6 shows calculated curves for $K_{\mathrm{s}}$ $=1.1 \times 10^{6} \mathrm{M}^{-1}(10,14)$ and several values of $K_{\mathrm{f}}$ and experimental data for $\alpha \beta-\alpha \beta^{\prime}$ mixtures with a total spectrin concentration of $1 \mathrm{mg} / \mathrm{ml}$. The scatter represents experimental error, which we have so far been unable to reduce. The effect of diluting $\alpha \beta$ with buffer, instead of with an identical volume of $\alpha \beta^{\prime}$ is shown for comparison.

The results leave no room for doubt that the $\alpha \beta^{\prime}$ dimers do indeed enter into the association reaction, and are consistent with a value of $K_{\mathrm{f}}$ close enough to that found for the univalent $\alpha$-chain fragment $\left(0.5 \times 10^{6} \mathrm{M}^{-1}\right)(10)$, and therefore to that expected if one binding site has been eliminated in the abnormal spectrin. The normal $(\alpha \beta)$ fraction of the spectrin, obtained by Sepharose chromatography of the low-temperature extract, behaves in respect of self-association like the spectrin from normal cells.

It can be seen from the calculated curves that in an equilibrium system in solution the introduction of $30 \%$ of univalent spectrin causes only a relatively small reduction in the proportion of tetramer in the system. This proportion is lower at higher spectrin concentration. Thus at a very high concentration $(90 \mathrm{mg} / \mathrm{ml}$, for example), at which the proportion of tetramer in normal spectrin at $30^{\circ} \mathrm{C}$ would be some $95 \%$, the replacement of $30 \%$ of the normal $(\alpha \beta)$ spectrin by $\alpha \beta^{\prime}$ would reduce the proportion of tetramer to only $\sim 90 \%$.

Rates of extraction. As a further demonstration that the proportion of spectrin dimer in the low-temperature extract 


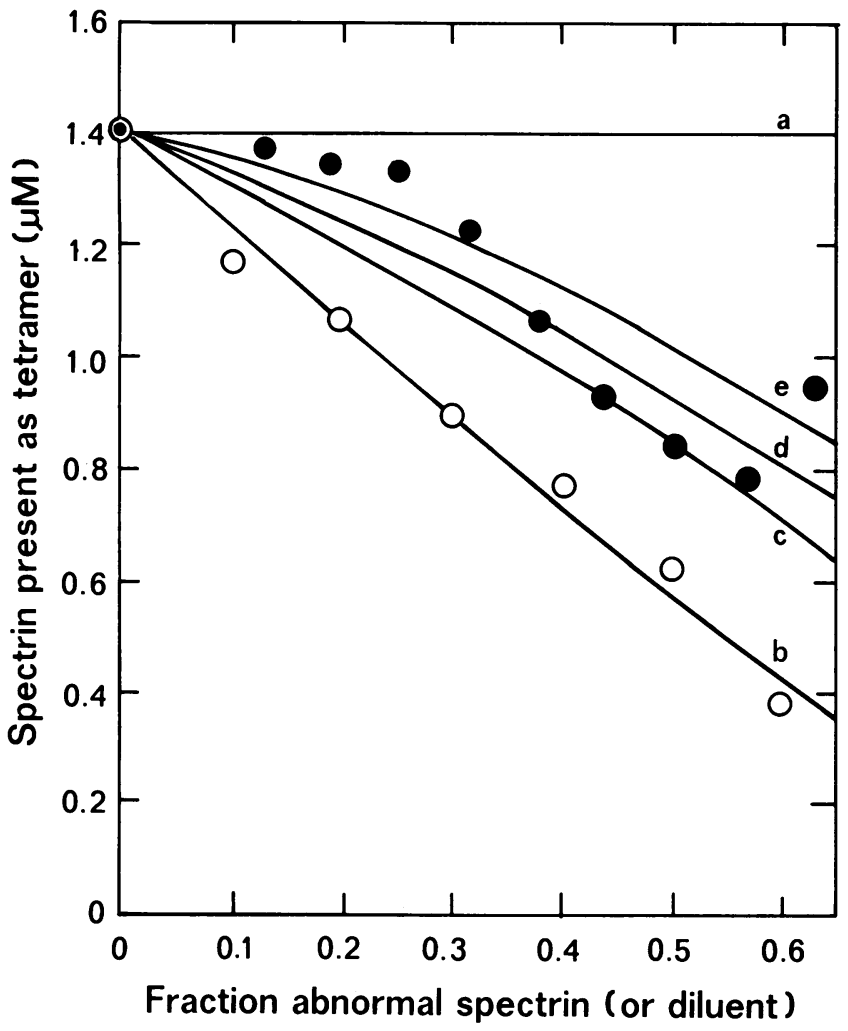

Figure 6. Self-association of normal and abnormal spectrin at $30^{\circ} \mathrm{C}$. The equilibrium tetramer concentration is shown as a function of the proportion of abnormal $\left(\alpha \beta^{\prime}\right)$ spectrin in the mixture $(\bullet)$, for a constant total spectrin concentration of $1.0 \mathrm{mg} / \mathrm{ml}$. In the control, which contains only normal spectrin (o), the dilutions were made with isotonic buffer in place of abnormal spectrin. The curves are calculated: tetramer concentration if $(a) \alpha \beta^{\prime}$ is functionally equivalent to $\alpha \beta$ (i.e., can form homo- and hetero-tetramers); $(b) \alpha \beta^{\prime}$ can enter into no associations (i.e., does not affect the behavior of the normal spectrin); (c)-(e) $\alpha \beta^{\prime}$ can form heterotetramers, $\alpha_{2} \beta \beta^{\prime}$, but no homotetramers. The normal self-association equilibrium constant, $K_{\mathrm{s}}$, was taken as $1.1 \times 10^{6} \mathrm{M}^{-1}$, and the association constant $K_{\mathrm{f}}$ for the formation of the heterotetramers as $0.5 \times 10^{6}(c), 0.75 \times 10^{6}(d)$, and $1.1 \times 10^{6}(e) \mathrm{M}^{-1}$. Note that in the case of normal spectrin alone the data should adhere to curve $(b)$. Concentration is expressed in molarity, referred to dimer molecular weight.

from the abnormal cells reflects the state in the cell, the relative rates of extraction of the normal and abnormal spectrins at $4^{\circ} \mathrm{C}$ were determined. The results are shown in Fig. 7. After $6 \mathrm{~h}$ the extract from normal cells contains only $\sim 2 \%$ of dimer. That from the cells of the patients contains $70-80 \%$ of dimer. Of this dimer some $95 \%$ contains the truncated $\beta$-chain. With increasing time of extraction the proportion of the abnormal spectrin falls and eventually approaches the fraction of this spectrin $(\sim 30 \%)$ in the cells, as revealed by SDS-gel electrophoresis. As a control we used normal ghosts, in which the spectrin had been largely dissociated to the dimer by incubation at $35^{\circ} \mathrm{C}$ in a medium of ionic strength $0.01 \mathrm{M}$. As Liu and Palek (22) showed, spectrin is not appreciably extracted in these conditions, but the low salt concentration permits conversion of a large proportion of the spectrin to dimers. On extracting the membranes thus treated at $4^{\circ} \mathrm{C}$, a much more rapid rate of release of spectrin was observed (Fig. 7) and the rapidly liberated spectrin was predominantly dimeric, showing

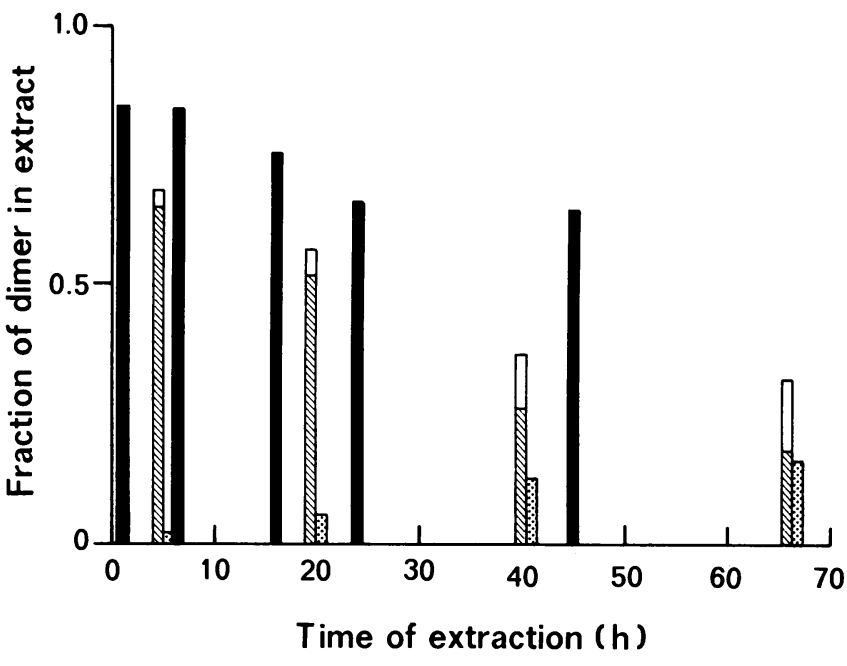

Figure 7. Rate of extraction of dimers, relative to tetramers, and of normal and abnormal $\left(\alpha \beta^{\prime}\right)$ spectrin from membranes at $4^{\circ} \mathrm{C}$ in low ionic strength. The bars represent the proportion of the extracted spectrin (tetramer and dimer) that is in the form of dimers. The stippled bars refer to extraction from normal membranes, the black bars to extraction from membranes that had undergone prior exposure to $35^{\circ} \mathrm{C}$ at $10 \mathrm{mM}$ ionic strength (22), so as to convert a large proportion $(\sim 70 \%)$ of the spectrin to dimers. The total proportion of spectrin liberated from the membrane after $2 \mathrm{~h}$ is $\sim 50$ and $10 \%$, respectively, in these cases. The remaining results refer to cells from the patient: the dimer in these cases consists predominantly of the abnormal type ( $\left.\alpha \beta^{\prime}\right)$ shown cross-hatched; the unshaded part is normal $(\alpha \beta)$ spectrin dimer. The small increase in the proportion of dimer at very long extraction times is presumed to be due to proteolytic damage.

that dimers are preferentially lost from the membrane (as indeed is to be expected, since they are retained at only one end by the cytoskeletal junctions).

After very long periods in the extraction medium (several days) the proportion of dimer in the total extract from untreated cells in general rises again, presumably as a result of proteolytic damage (Fig. 7).

Binding to the membrane. In seeking an explanation for the almost complete lack of heterologous association of spectrin dimer in cells containing $\alpha \beta^{\prime}$ dimers, one possibility that must be considered is an increased steric freedom of the abnormal dimers. Such an effect would lead to an entropic stabilization of the dissociated (dimeric) state and could come about from failure of the abnormal spectrin to attach firmly to the membrane. The primary means of interaction with the membrane is through binding to ankyrin, which is itself attached to the transmembrane protein, band $3(21)$. The ankyrin binding site of spectrin is on the $\beta$-chain (24) and is separated from the end of the chain, at which self-association occurs, by a segment of molecular weight $\sim 20,000$ (25). The possibility therefore suggested itself that the deletion in the $\beta$-chain also eliminates the ankyrin binding site. Accordingly, mixtures of $\alpha \beta$ and $\alpha \beta^{\prime}$ spectrin obtained from the abnormal membranes, were incubated at various concentrations with spectrin-depleted inside-out vesicles from normal membranes. Binding was measured by the loss of free spectrin from the solution after pelleting the vesicles. Heat-inactivated vesicles were used as controls. Fig. 8 shows that there is no significant difference between the binding of normal and of abnormal 


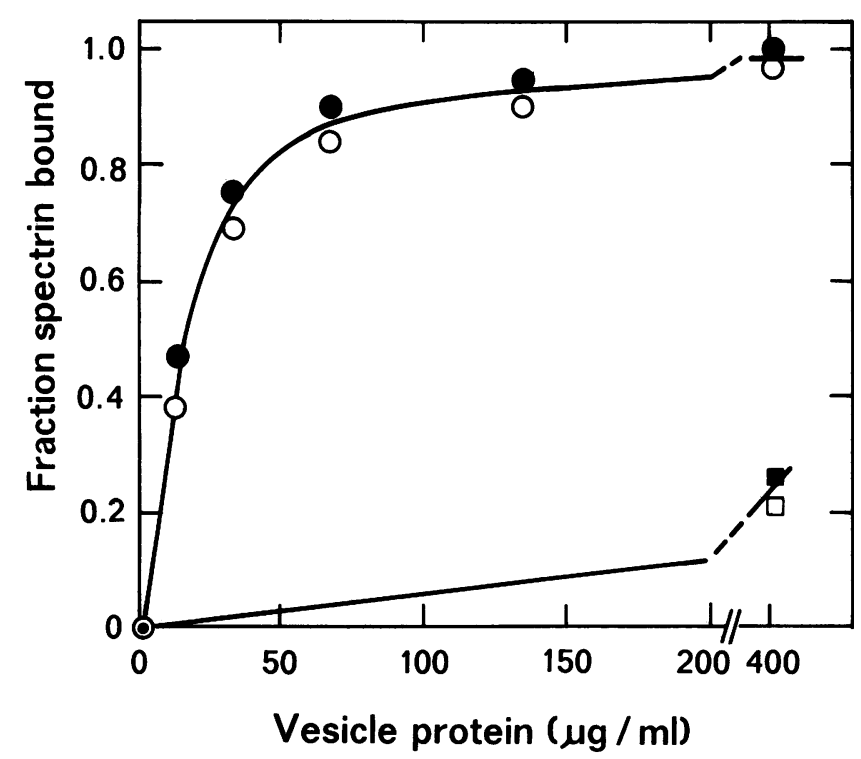

Figure 8. Rebinding of normal $(\alpha \beta)$ and abnormal $\left(\alpha \beta^{\prime}\right)$ spectrin dimers to spectrin-depleted vesicles. The vesicles were incubated with a mixture of the normal and abnormal spectrins in equal proportion at fixed spectrin concentration. Uptake of normal $(\bullet)$ and abnormal $(0)$ spectrin by the vesicles is shown. Corresponding controls for nonspecific binding $(a, 0)$ were performed with vesicles that had been heated for $10 \mathrm{~m}$ at $100^{\circ} \mathrm{C}$.

spectrins, and we conclude that the ankyrin binding site is not deleted in the truncated $\beta$-chains.

Reequilibration on the membrane. In an attempt to discover whether the exclusion of the univalent $\alpha \beta^{\prime}$ spectrin dimers from the tetrameric state is an intrinsic property of the membrane-associated system, mixed populations of $\alpha \beta$ and $\alpha \beta^{\prime}$ dimers were bound to spectrin-depleted membrane vesicles and were allowed to reassociate in situ by incubation at $30^{\circ} \mathrm{C} \mathrm{(3).} \mathrm{In} \mathrm{the} \mathrm{control,} \mathrm{which} \mathrm{contained} \mathrm{only} \mathrm{normal} \mathrm{spec-}$ trin, $\sim 55 \%$ of tetramer was formed on the membrane, as judged by reextraction at $4^{\circ} \mathrm{C}$, followed by gel electrophoretic analysis of the product. The incomplete self-association by contrast with the essentially complete reassociation of dimers, that occurs in intact ghosts (22), probably reflects the fact that the native cell contains only enough ankyrin to satisfy one of the two binding sites on each spectrin tetramer. Thus, when dimers are rebound the total number taken up is only half the number of dimer units that were present in the native cell (26).

With a mixture containing $50 \%$ of $\alpha \beta$ dimers, the total fraction of tetramer formed on the vesicles was about $15 \%$, of which only $15 \%$ was $\alpha \beta^{\prime}$. With a mixture containing $20 \%$ of $\alpha \beta^{\prime}$ dimers the proportion of tetramers rose to $50 \%$; no measurable proportion of $\alpha \beta$ spectrin could be detected in this fraction. Although the quantitative interpretation of these results poses some problems, they suggest that $\alpha \beta^{\prime}$ dimers on the membrane (by contrast with their behavior in solution) are largely precluded from entering into tetramer formation with normal dimers.

\section{Discussion}

We cannot state whether the mutation that has given rise to the abnormal spectrin is identical to those in the families in which truncated spectrin $\beta$-chains have previously been found $(6,7)$. The hematological consequences appear markedly different, since at least some of the members of the family described here have a severe hemolytic anemia, which necessitates periodic transfusions. These patients, moreover, display bizarre erythrocyte morphologies, in which poikilocytes predominate. By contrast, the cases described earlier $(6,7)$ were characterized by mainly smooth elliptocytes. The widely differing clinical symptoms and cell morphologies seen within the one family may indicate the presence of two mutations, one of which (not of course necessarily in spectrin) is clinically silent in the single heterozygote. Such an explanation has been advanced for the severity of the hemolytic anemia in hereditary pyropoikilocytosis, descending from a relatively symptom-free parent (1). In the case of the family depicted in Fig. 1, double heterozygosity would be compatible with the observed genetics only if both loci were on the same chromosome.

At the same time it seems to be generally the case that mutations that lead to impaired self-association of spectrin vary very widely in clinical severity (1). It does not appear, therefore, that the presence of a sizeable proportion of spectrin dimers in the cell must of itself have catastrophic consequences to the stability of the membrane. All such conclusions depend on the validity of the supposition that the composition of the spectrin in a low-temperature low-salt extract reflects its state in the cell. This appears to be fully justified by the high activation energy of the self-association process, which, as we have shown here, and has also been demonstrated for the interaction of the normal $\alpha$-chain C-terminal fragment with spectrin dimers $(8,9,10)$, applies equally to univalent interactions.

The small difference between the equilibrium constants for two-point and one-point associations is not necessarily surprising. It could be explained in terms of steric strain required to bring the second pair of sites into apposition once the first pair have bound, or, perhaps more likely, it arises from the internal association between the ends of the $\alpha$ - and $\beta$-subunits within the normal dimer, which Tyler et al. (27) have postulated to occur, on the basis of the appearance of the molecule in the electron microscope. This would stabilize the dimeric state and reduce the negative free energy of formation of tetramers. In the univalent species the existing binding site is not internally satisfied in this manner, and therefore only one internal interaction has to be broken in forming a heterotetramer, as against two for a homotetramer.

The single-point interaction is, as we have shown, strong enough that in a spectrin mixture of the composition prevailing in the abnormal cells, $\alpha_{2} \beta \beta^{\prime}$ heterotetramers should be abundantly formed, yet it is clear that essentially all of the $\alpha \beta^{\prime}$ units are dimeric. In searching for an explanation of this seeming paradox, it must first be recognized that simple massaction considerations are unlikely to apply to the self-association of spectrin on the membrane, for the law of mass action presupposes a diffusion-dependent dynamic equilibrium, and presumably diffusion of spectrin in the cytoskeleton is highly restricted. In the limiting case, any spectrin dimer, attached at its distal end to a multivalent junction point, consisting of actin and protein 4.1 (cf. references 28-30 for the structure of the lattice, as seen in the electron microscope), will be capable of binding to only one partner, projecting from a neighboring junction. In such a case, if the fraction of the abnormal spectrin is $f$, the proportion of the total spectrin capable of forming 
tetramers will comprise $(1-f)^{2}$ homotetramers $\left(\alpha_{2} \beta_{2}\right)$ and $2 f(1-f)$ heterotetramers $\left(\alpha_{2} \beta \beta^{\prime}\right)$. The remaining pairings, making up a proportion $f^{2}$, will involve only $\alpha \beta^{\prime}$ dimers, which will be incapable of interacting. With $30 \%$ of $\alpha \beta^{\prime}$ units, only $9 \%$ of the spectrin will be precluded from forming tetramers. Thus this apparently realistic model does not conform with the experimental observations, which would seem to show that no or very few heterotetramers are formed, and the tetramer population consists almost entirely of those (normal) dimer units (49\%) that should be apposed to another normal dimer unit.

The extent to which the normal self-association equilibrium in the cytoskeletal network favors the associated state must depend on the freedom of the spectrin chains to undergo segmental motion. The smaller the volume element within which the associating chain ends are confined the more favored will be the associated state. Impaired attachment to ankyrin would thus have afforded a rationale for reduced self-association. Since this can be excluded, the explanation must be of a different kind, e.g., $(a)$ total or partial segregation of the normal and mutant spectrins on the membrane. It is difficult to imagine any mechanism by which this could come about, although it could be conjectured that if assembly of the cytoskeleton proceeds by binding of tetramers to preexisting junctions, a cooperative accumulation of the species forming a greater proportion of tetramer, even by a small margin, could occur. According to the assembly principle developed by Lazarides and his co-workers (31), the unbound spectrin would then be eliminated by cytosolic proteases. This would explain why the product of the mutant spectrin gene makes up $<50 \%$ of the total spectrin in the cell. (b) Since, at least in avian cells, the spectrin seems to be delivered to the membrane as a tetramer (30), the possibility may be considered that $\alpha_{2} \beta_{2}$ tetramers enter the junction points, together with $\alpha \beta^{\prime}$ dimers. This would explain why $\alpha \beta$ dimers only are paired off, but such a mechanism would imply that no $\alpha_{2} \beta \beta^{\prime}$ tetramers are allowed to form between biosynthesis and cytoskeleton assembly. $(c)$ Even though the ankyrin binding site on the $\beta^{\prime}$ chain is intact, some other site of interaction with the membrane near the dimerdimer association sites might be missing. This could perhaps be a phospholipid binding region, such as is known to exist on spectrin $(32,33)$. However, since normal spectrin dimer, that has been generated in the ghost by treatment at low ionic strength, is preferentially released, just like the abnormal dimer, this explanation can probably be excluded. (d) Since we do not know how the spectrin self-association equilibrium on the membrane is regulated, we cannot exclude the possibility that even a small difference in the association constants measured in solution (a factor of two between those for one point and two-point interaction) could grossly affect the extent of association on the membrane, which may be much more strongly governed by such factors as segmental freedom. We favor the last of these explanations, on the grounds that the participation of the $\alpha \beta^{\prime}$ dimers in heterotetramer formation appears to be greatly restricted in the reconstituted system, using spectrin-depleted membrane vesicles. We cannot claim that these results are conclusive, because we have no definitive model for the analysis of the association process between dimers confined to the plane of the membrane. Two extreme cases may be considered. The first assumes that in the absence of network junctions on the inside-out vesicle membrane, the attachment of the spectrin to ankyrin is labile enough to allow dimers to diffuse over the membrane surface in search of thermodynamically optimal pairings. In this case the law of mass action could apply, and the $45 \%$ of dimers formed when normal spectrin is attached to the membane would represent an equilibrium proportion. If the association constant, $K_{\mathrm{s}}$, is the same as in free solution, we can calculate a pseudoconcentration of spectrin on the membrane. We may then use $K_{\mathrm{f}}$ and $K_{\mathrm{s}}$ as before to calculate the state of the $\alpha \beta-\alpha \beta^{\prime}$ mixtures on the membrane for the inferred effective concentration. For 50 and $20 \% \alpha \beta^{\prime}$ it emerges that the respective proportions of tetramer formed should be 0.32 and 0.46 , of which $\alpha \beta^{\prime}$ should make up 23 and $9 \%$, respectively.

The other extreme model assumes that the spectrin dimers cannot migrate around the membrane surface in search of partners, and that all dimers that can make contact with another functional dimer at their associating ends will form tetramers. In this case, the $50 \%$ tetramers formed by the normal spectrin controls represents the total of dimer-dimer contacts. In the mixtures containing 50 and $20 \% \alpha \beta^{\prime}, 0.41$ and 0.53 would be the fraction of spectrin forming tetramers and of these tetramers, $\alpha \beta^{\prime}$ spectrin should make up 33 and $16 \%$, respectively. The experimental proportions of $\alpha \beta^{\prime}$ in the tetramer fractions were well below the values predicted by either extreme model, and indeed the content was vanishingly small in the case of the spectrin mixture containing $20 \% \alpha \beta$. This strongly suggests, though it does not prove, that the exclusion of the univalent dimers from the tetramer fraction is an intrinsic thermodynamic attribute of the system on the membrane.

It may be remarked that amplification of a small difference in affinity of $\alpha \beta$ dimers for like and unlike dimers could result from an effect described by Georgi and Morrow (34): they reported that the binding of ankyrin is stronger to spectrin tetramers than to dimers. Conversely attachment of ankyrin to spectrin promotes conversion of dimers to tetramers. Thus a stronger tendency to associate, as between a pair of $\alpha \beta$ dimers, will be enhanced by the stronger binding of the associated form to ankyrin.

Our results suggest then that the dynamics of the cytoskeleton, in which at physiological temperature the spectrin dimer units can undergo rapid transitions between the associated and dissociated state, may be finely balanced, so that even a very small change in intrinsic affinity can grossly alter the overall state of association, and thus the mechanical properties of the structure.

\section{Acknowledgments}

We thank D. M. Shotton and S. Pittman for a generous gift of monoclonal antibodies, Mrs. U. Peters for technical help and E. Ungewickell and J. C. Pinder for help and advice on aspects of this work. In particular, we are grateful to the members of the family B., and especially to J.B., for their patience and generous willingness to give blood samples over a long period. Dr. Morris was supported by an MRC Training Studentship. This work received support from the Deutsche Forschungsgemeinschaft.

\section{References}

1. Palek, J., and S. E. Lux. 1983. Red cell membrane skeletal defects in hereditary and acquired hemolytic anemias. Semin. Hematol. 20:189-224.

2. Morrow, J. S., and V. T. Marchesi. 1981. Self-assembly of spec- 
trin oligomers in vitro. A basis for a dynamic cytoskeleton. J. Cell Biol. 88:463-468.

3. Liu, S.-C., P. Windisch, S. Kim, and J. Palek. 1984. Oligomeric states of spectrin in normal erythrocyte membranes: biochemical and electron microscopic studies. Cell. 37:587-594.

4. Lawler, J., S.-C. Liu, and J. Palek. 1982. Molecular defect of spectrin in hereditary pyropoikilocytosis. Alterations in trypsin resistant domain involved in spectrin self-association. J. Clin. Invest. 70:1019-1030.

5. Coetzer, T., and S. Zail. 1982. Spectrin tetramer-dimer equilibrium in hereditary elliptocytosis. Blood. 59:900-905.

6. Dhermy, D., M. C. Lecomte, M. Garbarz, C. Bournier, C. Galand, J. Gautero, C. Féo, N. Alloisio, J. Delaunay, and P. Boivin. 1982. Spectrin $\beta$-chain variant associated with hereditary elliptocytosis. $J$. Clin. Invest. 70:707-715.

7. Ohanian, V., J. P. Evans, and W. B. Gratzer. 1985. A case of elliptocytosis associated with a truncated spectrin chain. Br. J. Haematol. 61:31-39.

8. Morrow, J. S., D. W. Speicher, W. J. Knowles, S. J. Hsu, and V. T. Marchesi. 1980. Identification of functional domains of human erythrocyte spectrin. Proc. Natl. Acad. Sci. USA. 77:6592-6596.

9. Hanspal, M. and G. B. Ralston. 1981. Purification of a trypsininsensitive fragment of spectrin from human erythrocyte membranes. Biochim. Biophys. Acta. 609:133-139.

10. Shahbakhti, F., and W. B. Gratzer. 1986. An analysis of the self-association of human red cell spectrin. Biochemistry. 25:59695975.

11. Dacie, J. V. 1960. In The Haemolytic Anaemias: Congenital and Acquired. J. V. Dacie, editor. 2nd ed. Grune \& Stratton, Inc., New York. 82-150.

12. Tomaselli, M. B., K. M. John, and S. E. Lux. 1981. Elliptical erythrocyte membrane skeletons and heat-sensitive spectrin in hereditary elliptocytosis. Proc. Natl. Acad. Sci. USA. 78:1911-1915.

13. Pinder, J. C., and W. B. Gratzer. 1983. Structural and dynamic states of actin in the erythrocyte. J. Cell Biol. 96:768-775.

14. Dodge, J. T., C. Mitchell, and D. J. Hanahan. 1963. The preparation and chemical characteristics of hemoglobin-free ghosts of human erythrocytes. Arch. Biochem. Biophys. 100:119-130.

15. Ungewickell, E., and W. B. Gratzer. 1978. Self-association of human spectrin. A thermodynamic and kinetic study. Eur. J. Biochem. 88:379-385.

16. Laemmli, U. K. 1979. Cleavage of structural proteins during the assembly of bacteriophage T4. Nature (Lond.). 227:680-685.

17. Evans, J. P., A. J. Baines, I. M. Hann, I. Al-Hakim, S. M. Knowles, and A. Hoffbrand. 1983. Defective spectrin dimer-dimer association in a family with transfusion dependent homozygous hereditary elliptocytosis. Br. J. Haematol. 54:163-172.

18. Ungewickell, E. 1985. The 70-kd mammalian heat shock proteins are structurally and functionally related to the uncoating protein that releases clathrin triskelia from coated vesicles. EMBO (Eur. Mol. Biol. Organ.) J. 4:3385-3391.
19. Kam, Z., R. Josephs, H. Eisenberg, and W. B. Gratzer. 1977. Structural study of spectrin from human erythrocyte membranes. Biochemistry. 16:5568-5572.

20. Bennett, V., and D. Branton. 1977. Selective association of spectrin with the cytoplasmic surface of human erythrocyte plasma membranes. Quantitative determination with purified $\left[{ }^{32} \mathrm{P}\right]$ spectrin. $J$. Biol. Chem. 232:2753-2763.

21. Beutler, E., C. West, and K. G. Blume. 1976. The removal of leukocytes and platelets from whole blood. J. Lab. Clin. Med. 88:328333.

22. Liu, S.-C., and J. Palek. 1980. Spectrin tetramer-dimer equilibrium and the stability of erythrocyte membrane skeletons. Nature (Lond.) 285:386-388.

23. Bennett, V., and P. Stenbuck. 1980. Association between ankyrin and the cytoplasmic domain of band 3 isolated from human erythrocyte membranes. J. Biol. Chem. 255:6424-6432.

24. Calvert, R., P. Bennett, and W. Gratzer. 1980. Properties and structural role of the subunits of human spectrin. Eur. J. Biochem. 107:355-361.

25. Speicher, D. W., and V. T. Marchesi. 1984. Erythrocyte spectrin is comprised of many homologous triple helical segments. Nature (Lond.). 311:177-180.

26. Goodman, S. R., and S. A. Weidner. 1980. Binding of spectrin $\alpha_{2}-\beta_{2}$ tetramer to human erythrocyte membranes. J. Biol. Chem. 255:8082-8086.

27. Tyler, J. M., B. N. Reinhardt, and D. Branton. 1980. Associations of erythrocyte membrane proteins. Binding of purified bands 2.1 and 4.1 to spectrin. J. Biol. Chem. 255:7034-7038.

28. Shen, B. W., R. Josephs, and T. L. Steck. 1984. Ultrastructure of unit fragments of the skeleton of the human erythrocyte membrane. J. Cell Biol. 99:810-821.

29. Byers, T. J. and D. Branton. 1985. Visualisation of the protein associations in the erythrocyte membrane skeleton. Proc. Natl. Acad. Sci. USA. 82:6153-6157.

30. Nelson, W. J., and E. Lazarides. 1985. Post-translational control of membrane-skeleton (ankyrin and $\alpha \beta$ spectrin) assembly in early myogenesis. J. Cell Biol. 100:1726-1735.

31. Liu, S-C., L. H. Derick, and J. Palek. 1987. Visualization of the hexagonal lattice in the erythrocyte membrane skeleton. J. Cell Biol. 104:527-536.

32. Mombers, C., J. De Gier, R. A. Demel, and L. L. M. Van Deenen. 1980. Spectrin-phospholipid interaction. A monolayer study. Biochim. Biophys. Acta. 603:52-62.

33. Bonnet, D., and E. Begard. 1984. Interaction of anilinonaphthoyl labelled spectrin with fatty acids and phospholipids: a fluorescence study. Biochem. Biophys. Res. Commun. 120:344-350.

34. Georgi, M., and J. S. Morrow. 1985. Spectrin oligomerization and ankyrin binding are allosterically coupled. J. Cell Biol. 101:286a. (Abstr.) 